\title{
Langue des signes et administration de la justice: le cas des Seychelles
}

\author{
Lingua de sinais e administração juridica: o caso de \\ Seychelles
}

\author{
Monique GENDROT \\ Pôle LSF/Institut National des Jeunes Sourds de Paris (INJS)
}

\begin{abstract}
RÉSUMÉ: Jusqu'en 2013-2014, les Seychelles sont un pays où aucun citoyen sourd ne pouvait être poursuivi ou poursuivre en justice, faute d'une accessibilité en langue des signes. L'article montre comment, sous l'impulsion d'un programme des Nations-Unies, s'est mise en œuvre la formation puis la constitution d'une équipe mixte interprètes langues vocales du pays/langue des signes seychelloise et médiateurs sourds. Il s'attache à exposer les problématiques rencontrées, dont entre autres une langue des signes en danger, non encore renseignée via un dictionnaire qui lui permette d'être reconnue, puis décline les solutions et les stratégies développées afin de les contourner, pour ensuite conclure sur les objectifs atteints.
\end{abstract}

MOTS-CLÉ: Justice. Interprète. Langue de Signes. Seychelles. Médiateur sourd. Schématisation.

RESUMO: Até 2013-2014, as Seychelles são um País onde nenhum cidadão conseguia ser processado ou processar na justiça, por falta de acessibilidade em língua de sinais. O artigo mostra como, sob o impulso de um programa das Nações Unidas, implementou-se a formação e, em seguida, a constituição de uma equipe mista de intérpretes em línguas vocais do país/língua de sinais de Seychelles e mediadores surdos. Expomos as problemáticas encontradas, dentre outras, a de uma língua de sinais em risco, que ainda não foi registrada via dicionário que permitisse o seu reconhecimento, declinamos as soluções e as estratégias desenvolvidas afim de contorna-las, para posterior conclusão sobre os objetivos atingidos.

PALAVRAS-CHAVE: Justiça. Intérprete. Língua de sinais. Seychelles. Mediador surdo. Esquematização. 


\section{Le « cas » des seychelles: preambule}

Les Seychelles sont un pays où, jusqu'en 2013-2014, aucun citoyen sourd ne pouvait être poursuivi ou poursuivre en justice, faute d'une accessibilité en langue des signes. Aucun ne pouvait être interpelé, faute de pouvoir lui signifier ses droits, et ce pourquoi sa conduite était répréhensible.

En 2012, un appel à projets lancé par les Nations Unies auquel répond l'Association of People with Hearing Impairment (A.P.H.I) et l'Institut National de Jeunes Sourds (I.N.J.S) de Paris permet la formation et la mise en œuvre d'une équipe mixte interprètes langues vocales du pays/langue des signes seychelloise (S.S.L) et médiateurs sourds.

\section{Les Seychelles: situation geographique}

Les Seychelles, sous sa forme longue : la République des Seychelles, forment un archipel de 115 îles et îlots au total. Elles se situent dans l'Océan Indien, au Nord-Est de Madagascar et à 1600 km à l'est du Kenya.

Les trois îles principales sont : Mahé, Praslin et La Digue. Notre mission s’est déroulée dans la première : Mahé dont la capitale est Victoria.

\section{Les Seychelles: situation linguistique}

Les Seychelles sont un pays francophone. En effet, au peuplement de l'archipel, le français était la seule langue, à laquelle s'ajoutait avec l'ère de l'esclavage le Créole - langue approximative du français. Et le français resta langue principale, pendant l'ère coloniale britannique pendant plus de cent ans et ce jusqu'à 1947, suite à plusieurs doléances du gouverneur britannique Sir Selwyn Clarke.

$\mathrm{Au}$ moment de l'Indépendance, la Constitution en 1976 prônait le trilinguisme équilibré entre français, anglais et créole. Celle de la Seconde République en 1979 reconnaissait le statut du créole, alors qu'il fallut attendre la Constitution de la 3ème République en 1993 pour que cette dernière accorde 
statut égal de langue officielle aux trois langues- l'anglais, le français et le créole. 1

Toutefois, aujourd'hui, dans la vie quotidienne, le français est très peu utilisé et son niveau se trouve au plus bas de l'histoire. À l'Assemblée Nationale, c'est le créole qui prime alors qu'au Palais de Justice, l'anglais prédomine comme «language of the court». L'espace médiatique et le monde des affaires restent dominés par l'anglais et le créole. Le français reste essentiellement présent dans la diplomatie et le tourisme.

\section{Les Seychelles: demographie generale et demographie de la population sourde et malentendante}

La population des Seychelles a été estimée à 88300 habitants au 1er janvier 2011.2 Parmi elle, l'effectif de la population sourde et malentendante est évalué, à titre provisoire, à environ 600 personnes, tous âges et types de surdité confondus. 3

\section{Programme d'accessibilite a la justice}

5.1 Historique du programme

Le programme a été initié suite à un appel à projets lancé par les NationsUnies auquel 1'Association of People with Hearing Impairment (A.P.H.I) et l'Institut National de Jeunes Sourds (I.N.J.S) de Paris ont répondu. Il a fait l'objet d'une convention entre les deux parties.

La première a été créée en 2005 et œuvre en faveur de l'accès à l'éducation, l'emploi, la culture, la santé et l'insertion sociale des enfants et personnes sourdes aux Seychelles.

\footnotetext{
${ }^{1}$ Mme Marie-Reine Hoareau, représentante de l'UNESCO à Victoria- Article «Semaine de la francophonie : le trilinguisme » Journal Seychelles Nation 28.03.2012.

2 www.diplomatie.gouv.fr/fr/dossiers

3 Association of People with Hearing Impairement - Victoria Market- Seychelles.
} 
Pour ce faire, elle a signé une première Convention Cadre de Partenariat (2011-2014) avec les autorités seychelloises et l'INJS de Paris en faveur d'un projet de Développement de la Langue des Signes Seychelloise (S.S.L) et ce, dans le cadre de la Convention de l'O.N.U (article 24b) signée le 30 Mars 2007 par le gouvernement seychellois.

La convention dont nous parlons à présent se distingue de la première puisqu'elle s'inscrit dans le cadre d'un programme des Nations-Unies (Small Grant Funding Programme United Nations ou U.N.D.P) auquel 1'A.P.H.I et l'I.N.J.S de Paris ont répondu. Elle obéit ainsi à l'article 9 de la Convention de l'O.N.U en faveur des « droits des personnes handicapées », signée en 2009 par le gouvernement seychellois. Elle a été rédigée dans les trois langues : anglais, français, créole, puis a été signée à Paris le 24 octobre 2012 et à Mahé le 6 novembre 2012.

Pour l'INJS de Paris, il a été créé en 1791 et a été le premier établissement public pour enfants sourds au monde. Il l'a été par la Révolution Française afin de poursuivre l'œuvre entreprise par l'Abbé de l'Épée dans le but de permettre aux sourds d'accéder à l'éducation et à la citoyenneté. Depuis cette époque, l'INJS de Paris a maintenu une tradition d'échanges et d'amitié avec des institutions et des personnes de nombreux pays.

Les deux parties : l'I.N.J.S de Paris et l'A.PH.I se sont donc retrouvées unies, de par leurs missions respectives, à travers cette convention dont l'objectif principal était l'accès aux cours de justice des Seychelles pour les citoyens sourds du pays, l'exercice et la défense de leurs droits.

\subsection{Calendrier des missions}

La formation a totalisé $180 \mathrm{~h} 00$ de formation, réparties en trois missions de $60 \mathrm{~h} 00$ chacune selon le calendrier suivant :

Mission 1 : du lundi 29 octobre 2012 au mardi 06 novembre 2012

Mission 2: du lundi 4 mars 2013 au vendredi 15 mars 2013 
Mission 3 : du lundi 29 avril 2013 au vendredi 10 mai 2013

5.3 Préparation préalable aux actions de formation

Notons-le d'emblée, interprète et formatrice de nationalité et de culture française, j'ai dû me former, en amont de mes missions, au système judiciaire en vigueur aux Seychelles, issu à la fois du système français pour le Code Civil et du système anglo-saxon pour le Code Pénal. J'ai pris connaissance de la Constitution qui régit le pouvoir judiciaire en place en lien avec le pouvoir exécutif. En particulier, pour la nomination des juges.

Je me suis également imprégnée de l'histoire du pays dont j'allais former les ressortissants, sachant que celui-ci avait subi l'esclavage et plusieurs vagues d'occupation (colonisation). Enfin concernant le versant linguistique, et pour répondre à l'usage des langues employées majoritairement par les apprenants, je me suis initiée, puis familiarisée avec le créole seychellois et la langue des signes seychelloise. Cette occasion m'avait déjà été donnée lors d'une première mission réalisée en 2012 aux îles Seychelles dans le cadre de la convention en faveur du Projet de Développement de la Langue des Signes Seychelloise signée entre l'Institut, l'A.P.H.I et le gouvernement seychellois. Ma mission consistait alors à former une équipe mixte de conteurs sourds en SSL et de conteurs créoles afin de mettre à l'écran de petits programmes télévisés de sensibilisation à la SSL, sur la chaine nationale de télévision seychelloise, la S.B.C. Je m'étais alors pénétrée de la culture du pays.

5.4 Contenu de la formation

La formation a alterné entre modules théoriques et modules pratiques avec l'appui des acteurs locaux: juges des différentes juridictions seychelloises, officiers de probation, avocats, policiers, interprètes de langues vocales anglais/créole employés dans les tribunaux... 
Les modules théoriques regroupaient :

1. l'historique et la définition du modèle judiciaire seychellois qui allie le modèle judiciaire anglo-saxon et le modèle judiciaire français (code civil) défini selon la Constitution en cours.

2. la connaissance de l'organisation judiciaire seychelloise, des différentes cours de justice et tribunaux qui la constituent et de leur composition respective. 4

3. la connaissance des différentes sentences liées aux cas au pénal et aux cas au civil selon les différentes juridictions, et selon qu'elles soient attribuées par le Chef Juge ou les Juges Puînés, ou bien par le Senior Magistrat ou le Magistrat.

4. l'étude des procédures judiciaires

5. l'étude de la Convention des Nations Unies relative aux Droits des Personnes Handicapées, en particulier les articles concernant les Droits des personnes sourdes et malentendantes.

6. le code de déontologie et de conduite professionnelle auxquels sont soumis les interprètes et les médiateurs dans les cours de justice. Double documentation française et américaine recueillie sur Internet.

7. la place, fonction et rôle d'un interprète en milieu judiciaire.

8. la place, fonction et rôle d'un médiateur sourd en milieu judiciaire. Documentation américaine recueillie sur Internet. En effet, en France et en Europe peu de médiateurs sourds professionnalisés interviennent dans les tribunaux, faute de formation. Cette dernière vient d'être récemment pensée et proposée.

Les modules pratiques regroupaient:

1. des visites à la Cour Suprême, à la Cour d'Appel, la Cour des Magistrats et aux différents tribunaux tels le Tribunal de l'Emploi et des Ressources Humaines, le Tribunal de la Famille. Les apprenants assistaient à des

\footnotetext{
${ }^{4}$ A noter : les Cours « hautes » : la Cour Suprême, la Cour d'Appel, la Cour des Magistrats dépendent du «judiciaire». En revanche, les tribunaux dépendent des Ministères : ainsi le Tribunal de la Famille (Ministère Social), Tribunal pour l'Emploi (Ministère de l'Emploi et des Ressources Humaines) etc. La Juvenile Court dépend du « judiciaire », tout en étant lié au Ministère Social.
} 
«cas » présentés en cours et devaient en faire un compte-rendu en langue des signes lors de leur retour en classe. Nous avons également visité le poste principal de la police à Victoria.

2. des invitations et des «causeries » en classe avec l'Attorney Général (Procureur Général en France), le Directeur Général au Secrétariat du Tribunal de l'Emploi (greffe en France), de deux interprètes, attachés à la Cour des Seychelles, d'un officier de probation du Ministère Social, d'un travailleur social de la Section Protection de l'Enfant et Légal, entre autres. À chaque fois, ces causeries étaient précédées d'une mise en commun, entre apprenants et formatrice, des questions à poser à l'invité. Elles étaient l'occasion de préciser et d'approfondir les missions de chacun dans l'organigramme du système judiciaire seychellois.

3. des simulations et captations vidéo de situations d'interprétation et de médiation. Elles se déroulaient en classe avec la majorité des invités cités précédemment. Elles étaient ensuite analysées et évaluées avec les apprenants et la formatrice.

Notons que toutes les visites, invitations, «causeries », simulations de situations d'interprétations avec des acteurs locaux de l'appareil judiciaire seychellois ont été autant d'occasions de faire connaître la mise en place d'une formation d'interprètes de langue des signes seychelloise/langues vocales du pays, et de médiateurs sourds dans le domaine judiciaire, avec à terme, la constitution d'un service à part entière, dévolu à cette activité.

\subsection{Ses apprenants}

$\mathrm{Au}$ départ de la formation, cette dernière regroupait quatre apprenantsinterprètes (toutes femmes) et quatre apprenants-médiateurs (trois femmes et un homme).

Parmi les premières, deux d'entre elles sont dentistes au sein d'un dispensaire, et l'une est enseignante au Ministère de l'Éducation. Elle a en 
charge, avec une collègue sourde, la première classe créée pour enfants sourds et ouverte le 7 octobre 2010 aux Seychelles, à l'école Au Cap (Mahé). La quatrième est présidente de l'association A.P.H.I et œuvre pour le bien-être des sourds aux îles Seychelles.

Parmi les seconds, excepté une étudiante inscrite à l'Université (Unisey), peu sont scolarisés puisqu'enfants ils n'ont pu bénéficier d'un enseignement spécifique à la surdité, faute de structure en place. Ils étaient pris en charge parmi les autres enfants présentant tout type de handicaps confondus. Toutefois, parmi eux et malgré une scolarité déficiente, une jeune femme a été formée à l'enseignement de sa langue naturelle, la SSL et professe, en binôme avec sa collègue sur la nouvelle classe pour enfants sourds, évoquée plus haut. Leur maîtrise du créole écrit reste parcellaire (expression et réception). Idem pour l'anglais.

\subsection{Les problématiques de terrain}

Interprète Français/Langue des Signes Française (LSF), si mon expérience m'a conduite à exercer au titre d'interprète dans les tribunaux français, puis à former de futurs interprètes Français/LSF à l'Université Lille3, puis l'Université de Rouen, nous l'avons noté, le système judiciaire seychellois fait référence au système anglo-saxon. La langue de la loi est l'Anglais et les procès sont rendus dans cette langue. D'où la présence systématique en Cours de Justice d'interprètes anglais/créole seychellois. En effet, la majorité des citoyens aux Seychelles ont une maîtrise très parcellaire de l'Anglais.

La langue des signes seychelloise (S.S.L) est en cours de renseignement. Son premier tome du dictionnaire est en cours de réalisation. C'est Mr Alain Gébert, professeur de LSF à l'INJS de Paris, inscrit en Master de Lexicologie à Lille3 qui est chargé de mission à ce sujet. La réalisation et diffusion de ce premier tome amèneront à terme à la reconnaissance officielle de la SSL. Il est un enjeu essentiel des objectifs à atteindre au titre de la Convention de 
Partenariat signée entre le gouvernement seychellois, l'INJS de Paris et l'A.P.H.I concernant le Développement de la Langue des Signes Seychelloise. A ce stade du développement de cette langue, c'était ainsi relever la gageure de mettre en place une formation relative au domaine judiciaire sans que le lexique en langue des signes du pays n'ait encore été renseigné.

Pourtant nous nous trouvions face à cette interrogation:

«Devions-nous attendre que la langue des signes seychelloise soit terminée d'être renseignée ? Qui plus est dans le domaine judiciaire ? Avant de pouvoir rendre accessibles les cours de justice et les tribunaux aux citoyens sourds des Seychelles ? Avant qu'ils puissent exercer et défendre leurs droits ?»

Nous avons répondu «non » et avons apporté les solutions suivantes.

\subsection{Les solutions apportées}

Durant les quatre mois précédant ma mission, outre les moyens mis en œuvre pour m'approprier la Constitution et le fonctionnement de l'administration judiciaire des Seychelles, j'ai lu quotidiennement le journal en ligne Seychelles Nation, en relevant tous les articles concernant cette administration et les « cas » rapportés en Cours. Ceci afin d'étudier avec les futurs apprenants des mises en situation concrètes et de les familiariser à leur tour à la justice de leur pays et à son actualité.

Lors des visites, «causeries », et durant la formation je me suis donc employée à utiliser le créole seychellois et la langue des signes locale. Je m'étais familiarisée à ces deux langues lors de deux précédentes missions en janvier 2007 et juillet 2012. Concernant le lexique appliqué au domaine judiciaire, je 1'ai appris dans les deux langues employées dans les Cours de Justice des Seychelles, à savoir : l'anglais et le créole.

Nous avons constitué une équipe mixte apprenants interprètes et apprenants médiateurs afin de répondre à la double problématique d'une langue des signes non encore formellement renseignée et au tout début de son 
enseignement auprès du public entendant. Les premiers ont de maitriser le créole et l'anglais, mais pas suffisamment la langue des signes. Les seconds, formés et professionnalisés seraient à même de s'adapter aux justiciables sourds en cas de difficultés d'interprétation (compréhension et expression) rencontrées par leurs collègues interprètes sur le terrain.

Enfin, l'apport de nouvelles connaissances et l'assimilation de nouvelles notions par les apprenants médiateurs, tout au cours de leur formation à l'administration judiciaire de leur pays, ont été autant d'occasions de créer des «néologismes» (nouveaux signes) dans leur langue naturelle et, ainsi de l'enrichir.

Par ailleurs, nous avons employé massivement l'outil «schématisation ». En effet, celle-ci répond à plusieurs fonctions :

- Elle permet la «déverbalisation» des textes écrits en anglais ou en créole sous la forme de représentations mentales éminemment visuelles. Il s'agit de transformer et d'organiser l'information originale (texte) dans le sens d'une schématisation et d'une abstraction de ses traits principaux. Pour ce faire, cette dernière recourt à un ensemble suffisant de traits visuels pertinents, nécessaires à sa lecture, à sa mise en espace et par là même à sa mise en discours. Elle préfigure l'interprétation en langue des signes qui, elle aussi s'inscrit de fait dans l'espace et signifie concepts et objets par des traits visuels et saillants.

- Elle respecte la structure grammaticale de la langue des signes. En effet, en transposant un univers en 3D sur un plan, elle renvoie à une grammaire de l'image et obéit de fait à la structure de la langue des signes inscrite dans un espace de signation en 3D.

- Elle est utilisée comme support de cours pour aborder l'administration judiciaire et les «cas » jugés dans les différentes juridictions. Elle met à égalité les apprenants interprètes et les apprenants médiateurs. Elle est employée systématiquement en cours par la formatrice au tableau, parallèlement à la langue des signes renseignée sur place par les apprenants médiateurs. Elle l'est ensuite par tous les apprenants comme «médiation» principale à l'échange des 
connaissances: notions juridiques, organisation judiciaire, lexique anglais et créole spécifique au domaine de la justice. Elle participe de fait à l'accès aux idées et aux concepts en les et les renvoyant les unes aux autres avec un système de flèches.

- Elle sert de support lors des contrôles des connaissances et elle pallie là encore au peu de maitrise de la langue écrite par les apprenants médiateurs.

- Elle garde une trace « écrite » des notions et « cas » abordés en cours. À l'issue de ces derniers, les schématisations réalisées sont systématiquement photographiées et conservées dans des fichiers. Ou bien prises en note sur place par les apprenants. Elles participent de fait à la mémorisation.

- En l'absence d'un lexique en langue des signes non encore renseigné ou bien non encore émergé, elle laisse toute latitude aux locuteurs sourds pour le faire. En effet, dans la démarche de respecter la langue des signes locale, issue de la culture même du pays et de ses représentations, il n'était pas question pour nous que j'emploie et introduise la langue des signes française (LSF). Nous pensions ainsi au programme de Développement de la langue des signes seychelloise qui lie le gouvernement seychellois, l'APHI et l'INJS de Paris. Notre programme s'il concernait l'accessibilité à la justice pour les citoyens sourds n'en a pas moins servi la langue des signes locale et son enrichissement. Il s'est inscrit ainsi dans une visée linguistique du projet de développement de la langue.

- Enfin, elle sert de "prompteur» à l'occasion de conférence publique telle celle organisée le samedi 27 avril avec pour thème le Droit des Personnes Handicapées établi selon la Convention de l'O.N.U. 
Figure 1: Exemples de schématisations produites par les apprenants
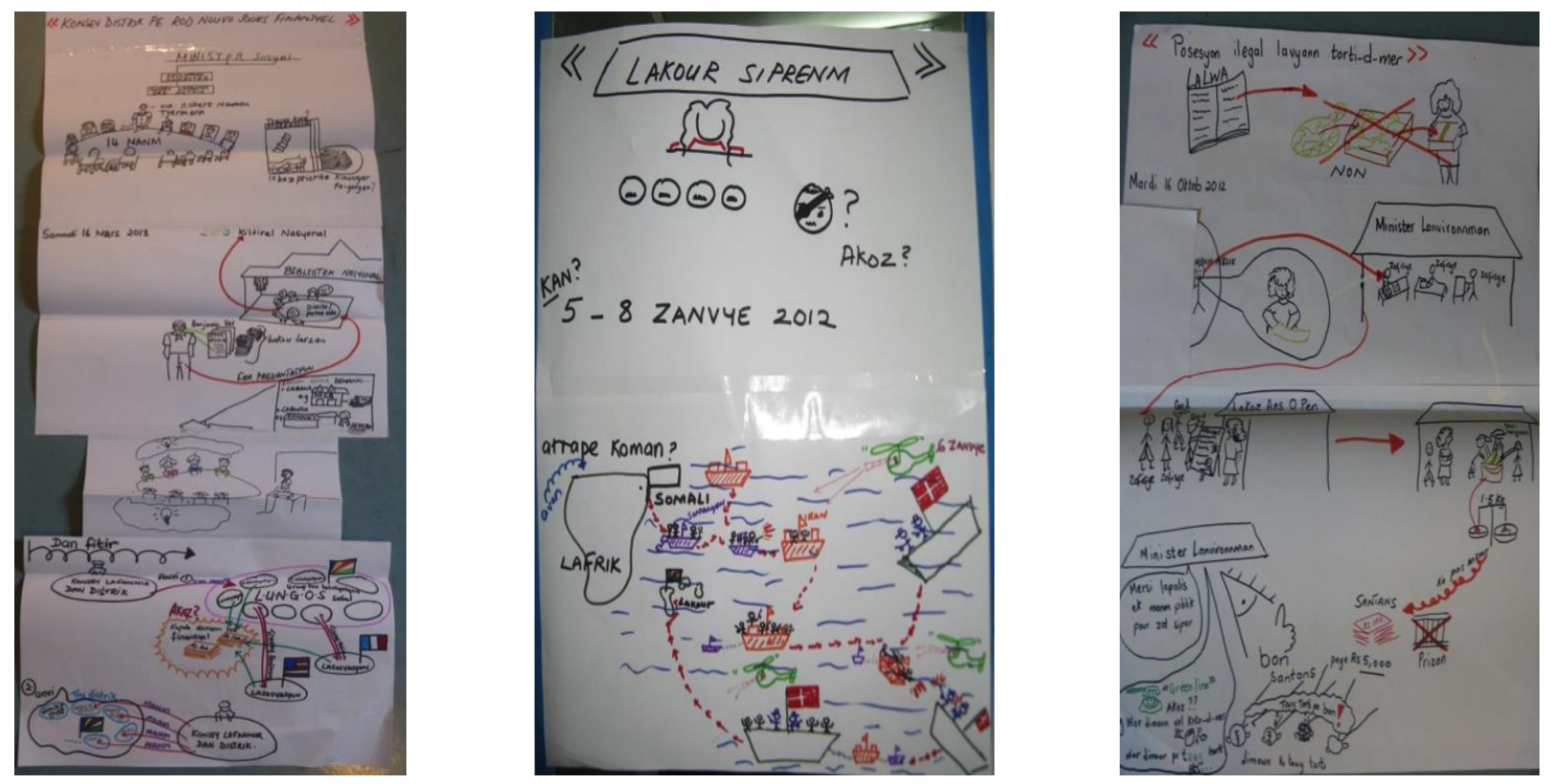

Source : Mission seychelloise, 2013.

\section{7 Les objectifs atteints}

1- Une découverte et une connaissance approfondie de l'administration judiciaire de leur propre pays par les apprenants interprètes et médiateurs

2- Une première étape vers une formation professionnalisante des interprètes et médiateurs aux Seychelles. En effet, si nous nous reportons à la situation locale des interprètes attachés aux Cours de Justice et aux tribunaux seychellois, il n'existe pas de formation formelle à l'instar de celle que nous avons initiée là-bas. Les renseignements recueillis auprès de deux interprètes attachés à la Cour des Seychelles sont les suivants :

- L'obtention du certificat A Level (baccalauréat en France) est obligatoire pour se porter candidat au poste d'interprète.

- Le recrutement s'effectue par voie de presse.

- Aucune connaissance spécifique relative à l'administration judiciaire du pays n'est exigée.

- Le choix des candidats au poste d'interprète s'établit à partir d'exercices de simulation de situations d'interprétation qui leur sont proposés. Ensuite, les lois et le Droit sont acquis, sur le terrain, au cours de la pratique de leur métier.

- Une fois recruté, le tout «jeune » interprète est «mis au travail » de la façon suivante: il effectue d'abord une période de 3 semaines d'observation, avant 
d'exercer sous la supervision d'un interprète confirmé. Il pratique d'abord dans les juridictions les plus «basses » tel le Tribunal de la Famille, le Tribunal pour l'Emploi et les Ressources Humaines, la Cour des Magistrats, et ce durant une période de 12 à 15 mois, avant de se voir attribuer, l'interprétation des audiences à la Cour Suprême. La raison d'une telle progression suivant le rang des tribunaux et des Cours de Justice en est l'emploi des langues lors des audiences. Les juges et avocats s'expriment en créole dans les premiers. Le juge en établit un compte-rendu en Anglais qu'il rédige à haute voix lors de l'audience. En revanche, les juges de la Cour Suprême s'expriment uniquement en anglais, et les débats avec les avocats également. En effet, seuls 3 juges de la Cour Suprême sont Seychellois. Les autres sont ressortissants de pays étrangers comme l'Angleterre, les pays africains, l'Inde... Ils ignorent le créole. Tout est traduit en créole par les interprètes. Toutefois, ces derniers sont tenus de signer le compte-rendu de l'audience enregistré et tapé dans un bureau attenant au tribunal. D'où une plus grande responsabilité à reconnaître ce compte-rendu comme l'expression de la vérité des débats.

- Aucun code éthique, ou de conduite, professionnel écrit n'existe sur lesquels puissent s'appuyer les interprètes et leurs usagers. Les règles sont tacites et la première en est la confidentialité. L'emploi d'un vocabulaire correct vient en second. Sinon les interprètes doivent se montrer solidaires entre eux. A ce sujet, notons l'intérêt porté par les deux interprètes invités pour un code de déontologie et de conduite écrit, à l'instar de ceux qui régissent la profession en Europe ou aux Etats-Unis. Ainsi, le modèle introduit lors de notre formation pourrait être étudié et appliqué, à terme, aux interprètes en activité dans les cours et tribunaux aux Seychelles.

3. Ils sont sortis certifiés à l'issue d'un contrôle de connaissances continu au cours des missions 2 et 3 .

4. Ils sont soumis à un Code Éthique et de Conduite clairement établi dans sa version écrite, auquel ils peuvent se référer, eux et leurs usagers.

5. Enfin, les interprètes et médiateurs formés dans le cadre du Programme UNDP d'accessibilité à la Justice pour les citoyens sourds des Seychelles ont eu à prouver leur capacité à exercer leur fonction au cours d'une année dite de «probation » 
(2013-2014) qui confirme leur aptitude à interpréter dans le respect du Code Éthique et Code de Conduite professionnelle auxquels ils sont soumis.

Nous apportons ici le témoignage de Mlles Shireen Guy et Shana David, respectivement interprète et médiatrice, formées dans le cadre du Programme UNDP d'accessibilité à la Justice. Il concerne la mise en œuvre de ce programme à l'issue des 3 sessions de formation.

À l'issue de la formation, et à l'instar de tout travailleur aux îles Seychelles, les interprètes et médiateurs, une fois certifiés, ont observé une période dite de «probation ». D'une durée d'un an, elle leur a permis de démontrer leur capacité à exercer leur fonction lors de prestations professionnelles, tout en appliquant le code éthique et de conduite professionnelle auxquels ils sont soumis.

Les dites prestations ont d'abord concerné des vacations effectuées au poste de police et dans les divers services du Ministère Social: Section Probation, Section Communautaire et Section Légal Protection de l'Enfance, avant d'exercer auprès des tribunaux.

Parallèlement à ces prestations, des «meetings » de sensibilisation aux Droits des Personnes Sourdes et à la mise en place du nouveau service d'interprètes et de médiateurs dans le domaine de la Justice sont organisés et animés (en créole et SSL) par la toute jeune équipe, en direction de la population sourde, des familles, des différents ministères et de l'administration judiciaire en général.

Afin de parfaire ses connaissances juridiques et améliorer le niveau de maîtrise de la Langue des Signes Seychelloise, voire créer des néologismes, l'équipe se réunit une fois par semaine dans les locaux de l'A.P.H.I. Ces rencontres servent également à réguler les activités et leur calendrier.

Enfin, à terme, au bout de l'année de probation écoulée, l'équipe espère pouvoir se constituer en association autonome en 2015, se détachant ainsi de l'A.P.H.I. Pour ce faire, elle devra se voir reconnue d'intérêt public par le Ministère Social et à ce titre, obtenir la rémunération de leur activité. Un comité sera créé au sein de cette dernière et composé entre autres d'un avocat, d'une personne sourde, et d'un membre de l'A.P.H.I. Ce comité aura à charge de recevoir les plaintes des usagers et d'y répondre en les arbitrant. 


\section{Conclusion}

Si nous mettons de côté notre vision occidentale des interprètes intervenant en Cours de Justice avec un niveau universitaire BAC+5 et une spécialisation au domaine judiciaire, si nous nous reportons au contexte local, et si nous nous référons au temps pris en France (1980-1999) avant d'inscrire la formation d'interprètes Français/Langue des Signes Française dans un cadre universitaire de niveau Master 2, notre formation, avec un total de 180 heures s'avère probante et efficiente. En effet, les apprenants ont bénéficié d'une formation théorique à l'administration judiciaire de leur pays. Ils se sont entrainés à des exercices de simulation de différentes situations d'interprétation auxquelles ils seront confrontés dans la pratique de leur métier, y compris les auditions au poste de police. Ils sont sortis certifiés et voient leur profession encadrée par un Code Ethique et de Conduite clairement établi et écrit auquel, eux et leurs usagers peuvent se référer. Enfin, une période d'un an, dite de «probation», les a confirmés dans l'exercice de leur fonction afin de rendre accessible aux citoyens sourds l'administration judiciaire de leur pays, à l'instar de tout autre citoyen de la République des Seychelles.

\section{RÉFÉRENCES}

Constitution of the Republic of Seychelles www.ilo.org/.../---ed.../wcms_127610.pdf

Seychelles: constitution de la République des Seychelles www.wipo.int/wipolex/fr/details.jsp?id=9115

Seychelles : Legal System www.bas.sc/seychelles-legal-system

\section{Convention des Nations-Unies relatives aux droits des personnes handicapées}

www.un.org/disabilities/documents/convention/convoptprot-f.pdf 